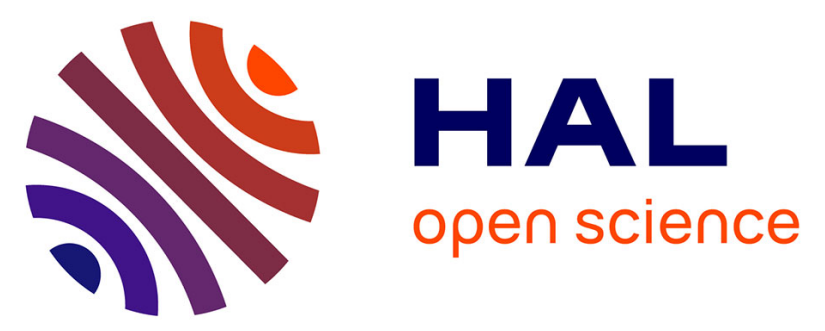

\title{
On the relevance of large scale pulsed-laser deposition: Evidence of structural heterogeneities in $\mathrm{ZnO}$ thin films
}

\author{
J Perrière, C Hebert, Nathalie Jedrecy, W Seiler, O Zanellato, X Portier, R
} Perez-Casero, E Millon, M Nistor

\section{- To cite this version:}

J Perrière, C Hebert, Nathalie Jedrecy, W Seiler, O Zanellato, et al.. On the relevance of large scale pulsed-laser deposition: Evidence of structural heterogeneities in $\mathrm{ZnO}$ thin films. Journal of Applied Physics, 2014, 116, pp.123502. 10.1063/1.4896379 . hal-01081606

\section{HAL Id: hal-01081606 https://hal.science/hal-01081606}

Submitted on 10 Nov 2014

HAL is a multi-disciplinary open access archive for the deposit and dissemination of scientific research documents, whether they are published or not. The documents may come from teaching and research institutions in France or abroad, or from public or private research centers.
L'archive ouverte pluridisciplinaire HAL, est destinée au dépôt et à la diffusion de documents scientifiques de niveau recherche, publiés ou non, émanant des établissements d'enseignement et de recherche français ou étrangers, des laboratoires publics ou privés. 




\section{Science Arts \& Métiers (SAM)}

is an open access repository that collects the work of Arts et Métiers ParisTech researchers and makes it freely available over the web where possible.

This is an author-deposited version published in: http://sam.ensam.eu

Handle ID: .http://hdl.handle.net/10985/8855

\section{To cite this version :}

J PERRIÈRE, C HEBERT, N. JEDRECY, W SEILER, O ZANELLATO, X PORTIER, R PEREZCASERO, E MILLON, M NISTOR - On the relevance of large scale pulsed-laser deposition: Evidence of structural heterogeneities in $\mathrm{ZnO}$ thin films - Journal of Applied Physics - Vol. 116, p.123502 - 2014 



\title{
On the relevance of large scale pulsed-laser deposition: Evidence of structural heterogeneities in $\mathrm{ZnO}$ thin films
}

\author{
J. Perrière, ${ }^{1,2}$ C. Hebert, ${ }^{1,2}$ N. Jedrecy,,${ }^{1,2}$ W. Seiler, ${ }^{3}$ O. Zanellato, ${ }^{3}$ X. Portier, ${ }^{4}$ \\ R. Perez-Casero, ${ }^{5}$ E. Millon, ${ }^{6}$ and M. Nistor ${ }^{7}$ \\ ${ }^{1}$ Sorbonne Universités, UPMC Univ Paris 06, UMR 7588, INSP, 4 Place Jussieu, F-75005 Paris, France \\ ${ }^{2}$ CNRS, UMR 7588, INSP, 4 Place Jussieu, F-75005 Paris, France \\ ${ }^{3}$ PIMM, UMR CNRS 8006 Arts et Métiers ParisTech, 151 Boulevard de l' hopital, 75013 Paris, France \\ ${ }^{4}$ CIMAP, CEA/CNRS UMR 6252/ENSICAEN/UCBN, 6 Boulevard du Maréchal Juin, 14050 Caen Cedex, \\ France \\ ${ }^{5}$ Departamento de Fisica Aplicada, Facultad de Ciencas, Universidad Autonoma de Madrid, \\ C/Francisco Tomas y Valiente 7, 28049 Madrid, Spain \\ ${ }^{6}$ Univ Orleans, UMR CNRS 7344, GREMI, 14 Rue Issoudun, F-45067 Orleans 2, France \\ ${ }^{7}$ National Institute for Lasers, Plasma and Radiation Physics, L22 PO Box MG-36, 77125 Bucharest, Romania
}

\begin{abstract}
Pulsed-laser deposition is known as a well-suited method for growing thin films of oxide compounds presenting a wide range of functional properties. A limitation of this method for industrial process is the very anisotropic expansion dynamics of the plasma plume, which induces difficulties to grow on large scale films with homogeneous thickness and composition. The specific aspect of the crystalline or orientation uniformity has not been investigated, despite its important role on oxide films properties. In this work, the crystalline parameters and the texture of zinc oxide films are studied as a function of position with respect to the central axis of the plasma plume. We demonstrate the existence of large non-uniformities in the films. The stoichiometry, the lattice parameter, and the distribution of crystallites orientations drastically depend on the position with respect to the plume axis, i.e., on the oblique incidence of the ablated species. The origin of these non-uniformities, in particular, the unexpected tilted orientation of the $\mathrm{ZnO}$ c-axis may be attributed to the combined effects of the oblique incidence and of the ratio between oxygen and zinc fluxes reaching the surface of the growing film.
\end{abstract}

\section{INTRODUCTION}

Pulsed-laser deposition (PLD) is a well suited deposition method for growing thin films of oxide compounds presenting either the physical properties of their bulk counterparts or new ones by changes in stoichiometry. ${ }^{1}$ Owing to the simplicity of the PLD process, oxide films can be deposited without sophisticated vacuum equipment, and the stoichiometric transfer of the target material to the substrate may usually be achieved, even in the case of complex materials. A limitation of PLD in industrial process is the very anisotropic character of ablation, which leads to severe nonuniformities at large scale in film thickness and composition, ${ }^{2-7}$ resulting in non-uniformities in film properties. $^{8}$ Actually, without any additional technologies to improve the thickness and composition uniformity, the substrate surface covered by a nearly homogeneous film is only about $1 \mathrm{~cm}^{2}$. A lot of efforts have been devoted to homogenize the film thickness and composition through various modifications of the PLD process: controlled laser rastering on large diameter targets coupled with target and/or substrate movements, ${ }^{1,9}$ inverse PLD ${ }^{10}$ or off-axis deposition, ${ }^{11}$ which in counterpart leads to a large decrease of the deposition rate. However, despite the important role of crystalline structure on oxide films properties, the specific aspect of structural uniformity of such large area films has not been widely studied.

The aim of this paper is to show how the anisotropy of the PLD process influences the crystalline structure of oxide films. In particular, we have tried to answer the following question: do the structural properties (crystalline phase, cell axis parameters, and texture) of the film depend on the position with respect to the centre of the ablation plume? Owing to the large number of studies on $\mathrm{ZnO}$ films, this material was chosen as a model system. Indeed, undoped or doped $\mathrm{ZnO}$ thin films can be used for a wide range of applications, and their properties drastically depend upon the structure and microstructure..$^{1,12-15} \mathrm{We}$ have investigated in details the stoichiometry and the crystalline characteristics (lattice parameters and domains orientation) of $\mathrm{ZnO}$ thin films as a function of position with respect to the central axis of the plasma plume, i.e. as a function of the oblique incidence of the ablated species. Unexpected tilted orientations of the $\mathrm{ZnO}$ c-axis were evidenced in the films, and their origin was related to the combined effects of the oblique incidence and of the oxygen and zinc fluxes reaching the substrate.

\section{EXPERIMENTAL}

Zinc oxide films were grown by PLD onto Si substrates covered by the native oxide ( $2 \mathrm{~nm}$ thick), or thermally oxidized $\mathrm{SiO}_{2}$ on $\mathrm{Si}$ substrates by using a frequency quadrupled $\mathrm{Nd}$ :YAG laser ( $5 \mathrm{~ns}$ pulse duration, $10 \mathrm{~Hz}$ repetition rate) in the experimental set-up described elsewhere. ${ }^{16}$ Pulses in the $0.5-2 \mathrm{~J} / \mathrm{cm}^{2}$ (with a spot diameter about $1 \mathrm{~mm}$ ) were used to ablate a pure $\mathrm{ZnO}$ ceramic target located at $5.5 \mathrm{~cm}$ in front of the substrate. This study is a part of a work on the correlation 
between the growth conditions and physical properties of zinc oxide films. To obtain a high density of carriers in the films, a low oxygen pressure was used during the growth to create oxygen vacancies, i.e., electrons donors. Film depositions were thus carried out under $10^{-6}$ mbar residual pressure, at room temperature (RT) and $500^{\circ} \mathrm{C}$.

The thickness and composition of the $\mathrm{ZnO}$ films were measured by Rutherford backscattering spectrometry (RBS) using the $2.5 \mathrm{MeV}$ Van de Graaff accelerator of the SAFIR IBA laboratory, University Pierre et Marie Curie. A ${ }^{4} \mathrm{He}^{+}$ ion beam with a $1 \mathrm{~mm}$ diameter was used for these experiments. Owing to the low RBS yield on light elements, the oxygen content of the film was only determined with a $4 \%$ precision, while the zinc content was determined with a $1 \%$ accuracy. To study the crystalline structure of the films, Xray diffraction (XRD) measurements were performed with a four circle goniometer (Philips Xpert MRD) using the $\mathrm{Cu} \mathrm{K} \alpha$ radiation. Symmetric Bragg-Brentano geometry and asymmetric XRD were used to determine the lattice parameters and texture of the zinc oxide film as a function of position with respect to the normal to the target. Cross sectional foils were prepared for transmission electron microscopy (TEM) observations by a standard mechanical procedure involving grinding, dimpling, and argon ion milling until electron transparency. The foils were observed by conventional TEM and high resolution transmission electron microscopy (HRTEM) using a FEG 2010 JEOL equipment operated at $200 \mathrm{kV}$. Image processing was performed using the commercial DigitalMicrograph software from GATAN.

\section{RESULTS}

The anisotropic character of the PLD method is schematically represented in the inset of Fig. 1. The ablation plume is centred on the normal to the target at the laser spot position. Owing to the plume expansion, the flux of incident species from the target to the substrate depends upon the position with respect to the plume central axis. In the inset, the angle $\alpha$ of which is directly related to the substrate

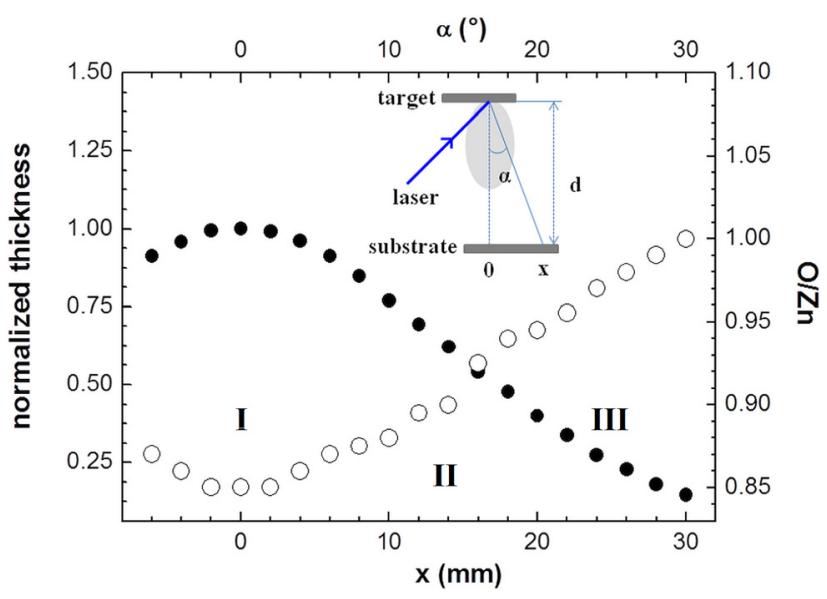

FIG. 1. Thickness and composition variation for a $\mathrm{Zn}$ oxide film grown by PLD at RT and $10^{-6} \mathrm{mbar}$, as a function of substrate position (the origin of position being the normal to the target at the laser spot). The corresponding incident angle for the species is also indicated. The schematic description of the anisotropic character of PLD is presented in inset. position $\mathrm{x}$ and distance $\mathrm{d}$ between the target and the substrate: $\operatorname{tg} \alpha=\mathrm{x} / \mathrm{d}$, has been defined as the "deposition angle." In this paper, this deposition angle $\alpha$ plays a major role on the film structure, and it has been used as the key parameter in the next figures instead of the x position. It follows from the anisotropy of the process that the film thickness is not uniform, as shown in Fig. 1, for a zinc oxide film grown at RT and $10^{-6}$ mbar. A bell shape curve of the normalized thickness (th/th $\mathrm{th}_{0}$ with $\mathrm{th}_{0}$ being the thickness for $\alpha=0$ ) is observed as a function of the deposition angle $\alpha$. Such an angular distribution of thickness has been usually observed for a lot of materials deposited by PLD ${ }^{2-7}$ and these curves have been interpreted in the frame of the Anisimov's model. ${ }^{4}$ The same kind of curve was observed in this work for zinc oxide films deposited by PLD at $500^{\circ} \mathrm{C}$.

Fig. 1 clearly demonstrates that the O/Zn composition ratio varies as a function of the substrate position. Such non uniform angular distribution of elements has been previously reported for multicomponent materials, i.e., in the PLD plume, the spatial distribution of the elements depends upon their mass in relation with gas scattering process. ${ }^{17-21}$ The studies concerning the angular distribution in oxide materials were generally not focused on the angular distribution of oxygen with respect to the cations. For oxygen, in addition to the difference of mass with the cations which can play a role, another problem is the high volatility of this element leading to large losses during PLD, which must be compensated by working under an oxygen pressure in order to obtain stoichiometric compounds. The ratio of fluxes of oxygen and cation governs the oxygen incorporation in the oxide films. ${ }^{22,23} \mathrm{It}$ can be deduced from Fig. 1 that for $\alpha=0$ (region I) where a largely oxygen deficient zinc oxide is formed, the growth conditions are highly reducing (i.e., cations flux largely higher than the oxygen flux). For increasing deposition angle (region II), the oxygen deficiency in the film decreases corresponding to an increase of the $\mathrm{O} / \mathrm{Zn}$ ratio of fluxes. A further increase of this ratio leads to the formation of stoichiometric $\mathrm{ZnO}$ in region III. Similar experiments carried out at $500{ }^{\circ} \mathrm{C}$ lead to a similar behavior attenuated by an increase of the oxygen incorporation in the film due to the high temperature. As a matter of fact, a $\mathrm{ZnO}_{0.9}$ was observed for $\alpha=0$.

$\mathrm{X}$-ray diffraction $\theta$-2 $\theta$ diagrams recorded for various $\alpha$ values (Fig. 2) show drastic structural non-uniformities. Three distinct regions can be defined. In the central region, i.e., zone $\mathrm{I}\left(-5^{\circ}<\alpha<5^{\circ}\right)$, a sole diffraction peak is present corresponding to the 002 diffraction line of a c-axis oriented (001) $\mathrm{ZnO}$ film. In the intermediate zone II $\left(5^{\circ}<\alpha<20^{\circ}\right)$, both the 002 and 101 peaks are present attesting of the polycrystalline like character in this zone. For larger oblique incidences, i.e., in zone III $\left(\alpha>20^{\circ}\right)$, a sharp 002 diffraction line is observed, while a slight contribution of the 101 diffraction line cannot be excluded. The inset in Fig. 2 represents the variation of the 002 peak intensity as a function of the angle of incidence of the species. The intensity of the 002 peak normalized to the film thickness is also presented in the inset, and shows that a higher texture is observed at large $\alpha$ values. The three zones are clearly evidenced in the inset, and rather abrupt transitions are observed between the different zones. 


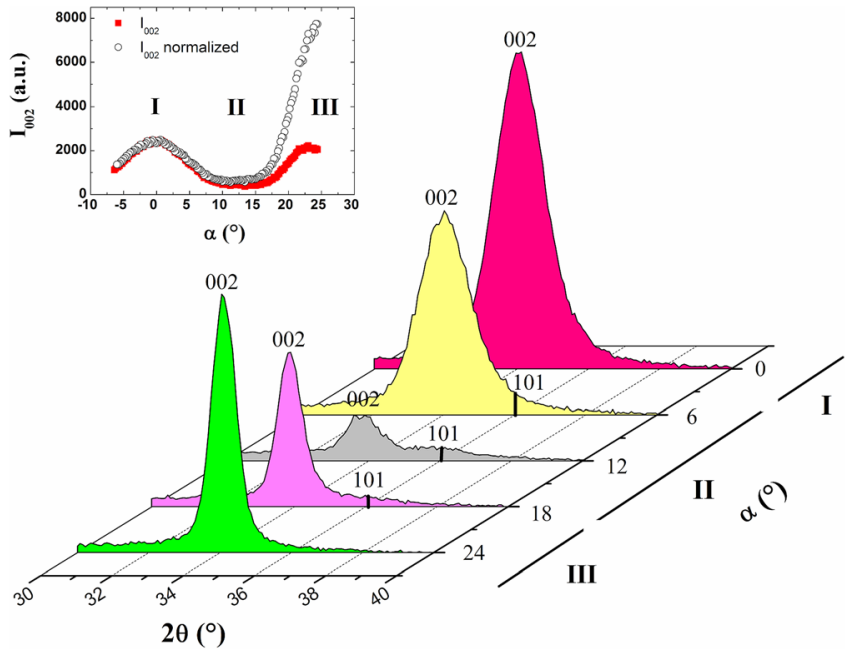

FIG. 2. X-ray diffraction diagram for a $\mathrm{Zn}$ oxide film grown at room $\mathrm{T}$ and $10^{-6}$ mbar as function of the angle of incidence of the species $\alpha$. The variations of the intensity (as recorded and normalized to the film thickness) of the 002 diffraction line of $\mathrm{ZnO}$ as a function of the incident angle of the species $\alpha$ is presented in inset.

The c-axis texture is usually predominant for $\mathrm{ZnO}$ films whatever the substrate and the growth method. ${ }^{24-26}$ This is related to the nature of the $(001) \mathrm{ZnO}$ planes and to the high growth kinetic along the [001] direction. ${ }^{26}$ In the wurtzite structure, the (001) planes are polar (either $\mathrm{Zn}$ or $\mathrm{O}$ atomic planes) and the surface free energy of such planes diverges, ${ }^{27}$ while the non-polar prismatic planes ((100) or (110)) have a lower surface free energy. In principle, the growth of $\mathrm{ZnO}$ films should thus occur with a prismatic plane parallel to the substrate. However, kinetics effects influence the film formation, and the very high growth rate of $\mathrm{ZnO}$ along the c-axis direction leads to a columnar growth, ${ }^{1,28}$ which is observed despite the unfavorable surface free energy of the (001) family planes.

The appearance of (101)-oriented $\mathrm{ZnO}$ crystallites in the zone II seems related to the evolution of the ratio of fluxes $(\mathrm{O} /$ $\mathrm{Zn}$ ) as a function of the position on the substrate. Indeed, it has been observed by sputtering ${ }^{29}$ as well as by PLD $^{30}$ that $\mathrm{Zn}$ rich conditions lead to pure c-axis texture, while increasing oxygen flux leads to the formation of mixed (001) and (101) textures. A change in texture associated with a change in the pressure of the ambient gas has already been observed in other systems ${ }^{16,31}$ and could be related to the change in surface free energy of the planes as a function of the conditions, ${ }^{32}$ i.e., surfactant effects related to oxygen rich growth conditions in the present case.

In addition to these micro-structural differences, the lattice parameter value was also found to vary as it can be deduced from Fig. 3. For films grown at RT, this parameter is higher than the bulk value $(0.5206 \mathrm{~nm})$, and decreases with the deposition angle. For a growth at $500^{\circ} \mathrm{C}$, this effect is very limited: one observes a very weak decrease towards the bulk value for increasing incidence angle. Moreover, on contrary to the growth at RT, the 101 diffraction peak is not observed for films grown at $500{ }^{\circ} \mathrm{C}$. The variations in the lattice parameter of zinc oxide PLD films are usually attributed to the presence of strain in the films, ${ }^{1,13}$ but the variation in oxygen composition as a function of position on the substrate (Fig. 2) may also play a role.

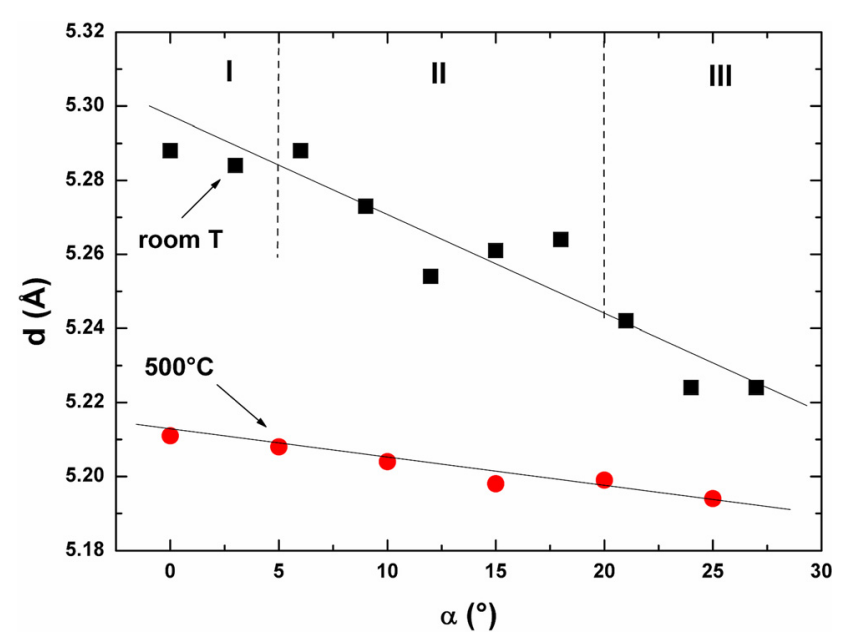

FIG. 3. c-axis parameter values as a function of the incident angle of the species $\alpha$ for films grown under $10^{-6}$ mbar and at room $\mathrm{T}$ and $500^{\circ} \mathrm{C}$.

The specific three distinct crystalline zones were further studied by transmission electron microscopy, and Fig. 4 shows the cross-sectional low magnification TEM images of the zinc oxide film at three different positions on the substrate. A sharp interface and a columnar growth of the film are observed in Figs. 4(a) and 4(c) corresponding, respectively, to the central region (zone I) and to the external zone (zone III). By contrast, no columnar structure appears in the intermediate region (zone II), confirming the polycrystalline nature of the zinc oxide film in this zone. The selected area electron diffraction (SAED) patterns (corresponding to an area of about $0.1 \mu \mathrm{m}^{2}$ ) below the TEM images are in good agreement with the XRD data. Indeed, elongated 002 spots due to an angle distribution of the crystallographic orientation of the columns are observed in the SAED patterns corresponding to zone I and zone III. This distribution covers an angle of about $27.5^{\circ}$ and $20^{\circ}$ for zones I and III, respectively.

Figs. 5(a) and 5(b) are high resolution TEM images from a cross section of region II. Fig. 5(a) shows a (101)-oriented $\mathrm{ZnO}$ crystallite close to the $\mathrm{SiO}_{2} / \mathrm{Si}$ substrate. The
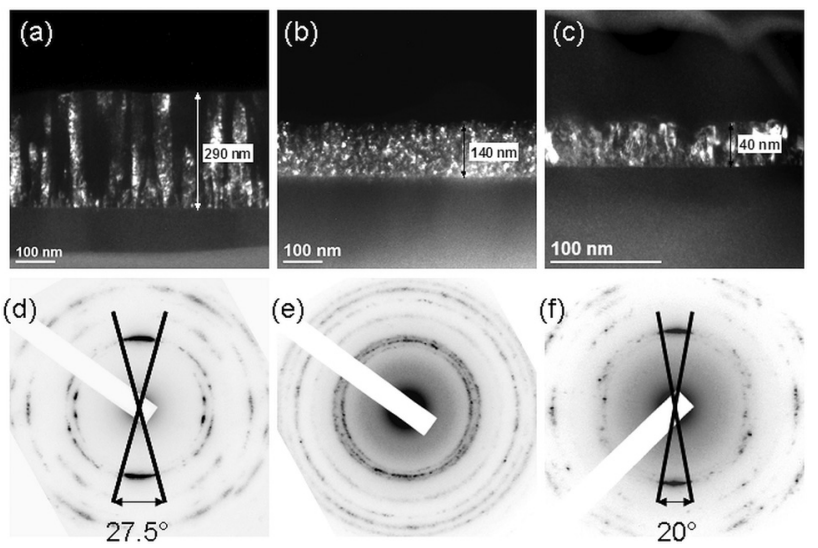

(e)

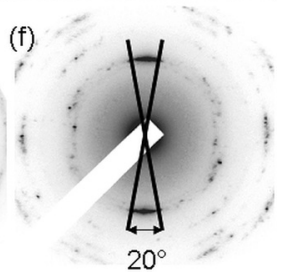

FIG. 4. Dark field TEM images of cross sectional views of three different regions called zone I (a), zone II (b), and zone III (c) in the text. Note the decreasing thickness value from zone I to zone III. The corresponding SAED patterns are reported in (d), (e), and (f) for zones I, II, and III, respectively. Clear (001) textures are observed for zones I and III. By contrast, this texture is not visible for zone II. 

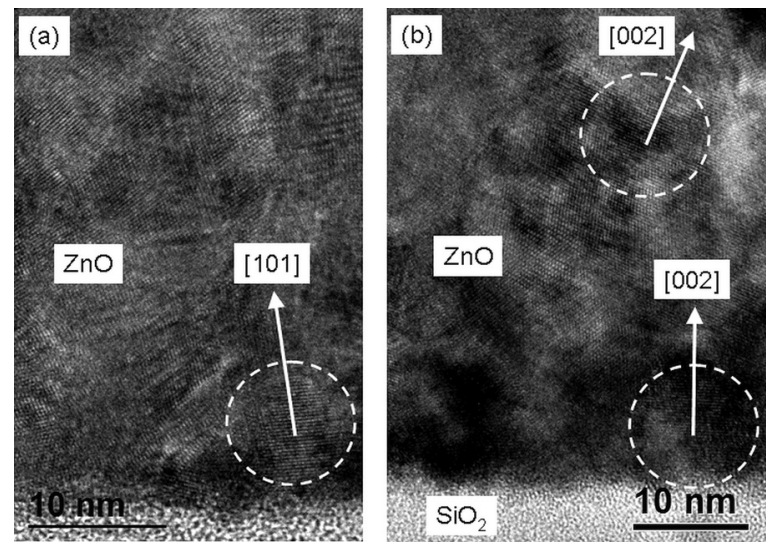

FIG. 5. HREM images taken in zone II and confirming the loss of the (001) texture in this region. The substrate/film interface is horizontal. The polycrystalline character of this zone appears with (101) and (001)-oriented grains in (a) and large variation in the (001)-oriented grains is present in (b).

polycrystalline nature of the zone II clearly appears via the various orientations of the $\mathrm{ZnO}$ crystallites. Fig. 5(b) shows typical region of zone II where the growth is no longer columnar but granular with grains randomly oriented in appearance. As an example, two [001] directions from two different grains are indicated and they appear clearly misoriented each other by an angle of about $20^{\circ}$.

Complementary information was obtained by 002 pole figure measurements. In such figures, the 002 poles are characterized by a declination $(\psi)$ and azimuthal $(\varphi)$ angle, which define the precise orientation of the (001)-oriented $\mathrm{ZnO}$ crystallites with respect to the normal to the substrate. Fig. 6(a) shows that under normal incidence $(\alpha=0)$, i.e., in zone I, a single broad 002 pole centered at $\Psi=0$ is observed meaning that the [001] axes are distributed symmetrically around the normal to the substrate. In the same way, in Fig. 6(c) obtained for $\alpha=25^{\circ}$, i.e., zone III, a single pole centered at $\Psi=0$ is also present. The comparison of Figs. 6(a) and 6(c) clearly confirms a better c-axis texture in zone III than in zone I.

In Fig. 6(b) corresponding to zone II $\left(\alpha=13^{\circ}\right)$, a welldefined single 002 pole is not present. On the contrary, a distribution of 002 poles is observed, looking like a fiber texture, approximately centered in the direction of incident species (indicated with an arrow). However, this distribution of 002 poles is not fully circular, and a large strengthening of the 002 pole distribution is present in the direction opposite to that of the incident species. Looking at the high values of the declination ( $\Psi$ around $75^{\circ}$ ), another semi-circular pole distribution (with a limited intensity) seems to be present in the direction opposite to the incident species.
To determine quantitatively the tilt of the $\mathrm{ZnO}$-axis with respect to the normal to the substrate ( $\beta$ angle) and its variation with the deposition angle $\alpha$, i.e. to determine the $\beta=\mathrm{f}(\alpha)$ variation, $\Psi$ scans for the 002 diffraction line were registered as a function of the position on the substrate, and are presented in Fig. 7. As expected from Fig. 6(a), under normal incidence a single broad peak centered at $\Psi=0^{\circ}$ is present. This broad peak corresponds to the wide angle dispersion of the [001] axis with respect to the normal already observed in the SAED (Fig. 4).

For increasing $\alpha$ values, this main peak moves in the direction opposite to the incident species, which is indicated on each $\Psi$ scan by a mark, i.e., the orientation of the [001] axis is located in the forward direction, as shown in an inset of Fig. 7. This is the reverse of the orientation classically reported in the case of the shadowing effect associated with oblique incidence deposition films. ${ }^{33-35}$ Actually, under an oblique angle deposition $\alpha$ at room temperature (low adatom mobility), due to the self-shadowing effect, a columnar growth is observed inclined towards the incoming flux of species. An empirical relationship between the incidence angle $\alpha$ and the inclination of texture $\beta " \operatorname{tg} \alpha=2 \operatorname{tg} \beta "$ has been previously reported for films grown by various methods. ${ }^{33-35}$ The reverse situation is observed here. The position of the maximum of the main peak in Fig. 7 measures the tilt $\beta$ of the c-axis, and its variation is presented in Fig. 8 . The angle $\beta$ is at first an increasing function of $\alpha$, but for $\alpha>20^{\circ}$ (end of zone II), the tilt of the c-axis decreases sharply and for $\alpha$ higher than $25^{\circ}, \beta$ is equal to zero meaning that a $\mathrm{c}$-axis texture is present for large oblique incidence.

In Fig. 7, in addition to the "central" (main) intense peak, two other low intensity peaks can be distinguished in the $\Psi$ scans recorded for $5^{\circ}<\alpha<20^{\circ}$. These $\Psi$ scans corresponding to zone II are presented with a logarithm scale in an inset in Fig. 7, allowing a better observation of the low intensity peaks. These smaller peaks are located at a roughly equal angular distance of the main peak, i.e., around $60^{\circ}$, and these two smaller peaks follow the variation of position of the main peak, meaning that a well-defined relationship exists between these peaks. Two points have to be taken into consideration to explain the origin of these low intensity peaks. First, (101)-oriented $\mathrm{ZnO}$ crystallites are present in zone II (and zone III) as evidenced by XRD analyses (Fig. 2 ), and second, the angle between the (001) and (101) planes in the $\mathrm{ZnO}$ wurtzite structure is $61.6^{\circ}$. As a result, assuming that the (001) and (101)-oriented crystallites in zone II are both inclined by $\beta$, the two low intensity peaks are due to the (001) planes of these (101) (and (10-1)) oriented crystallites tilted by an angle $\beta$. More detailed explanations of the origin
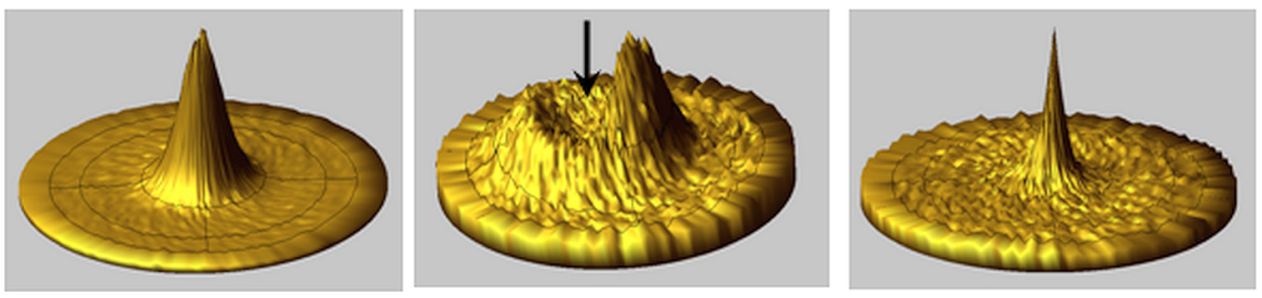

FIG. 6. Pole figure of the (002) $\mathrm{ZnO}$ planes for (a) $\alpha=0^{\circ}$, (b) $\alpha=13^{\circ}$, and (c) $\alpha=25^{\circ}$. 


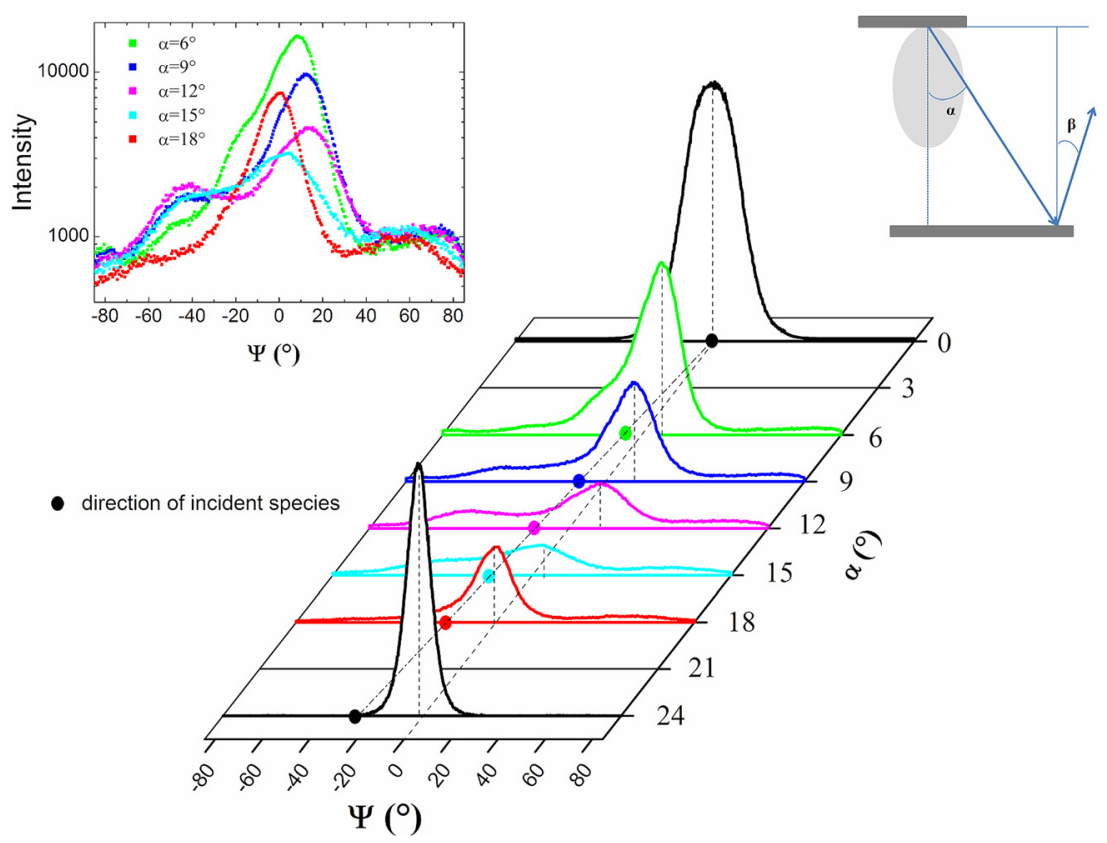

FIG. 7. $\Psi$ scan for the 002 reflection plane as a function of the incident angle of species $\alpha$ for a $\mathrm{ZnO}$ film grown at room $\mathrm{T}$ and $10^{-6} \mathrm{mbar}$. The $\Psi$ scans corresponding to the zone II are presented with a log scale in one inset. The other inset shows schematically the respective directions of the incident species and the tilted c-axis.

of these two low intensity peaks are given in the Appendix of this paper. Moreover, all these peaks are not symmetric, a broadening is observed on each peak, mainly in the direction of the incident species, meaning that a rather wide distribution of the tilt value exists for the (001) and (101)-oriented crystallites.

Finally, the influence of the substrate temperature was studied. This parameter plays an important role on the c-axis inclination, as it can be deduced from Fig. 9, which represents the $\Psi$ scans obtained for a film grown at $500^{\circ} \mathrm{C}$ and $10^{-6}$ mbar. These $\Psi$ scans show that contrary to the RT case, the c-axis is very close to the normal to the substrate. This is due to the high surface diffusion coefficient for the incident species at $500^{\circ} \mathrm{C}$. However, a clear deviation of a few degrees is observed in the $\beta=\mathrm{f}(\alpha)$ presented in the inset of Fig. 9, meaning that the structural quality of the $\mathrm{ZnO}$ films depends upon the incident direction of the species even at high $\mathrm{T}$. Interestingly, this slight deviation was found towards the direction of the incident species, as it is observed in the

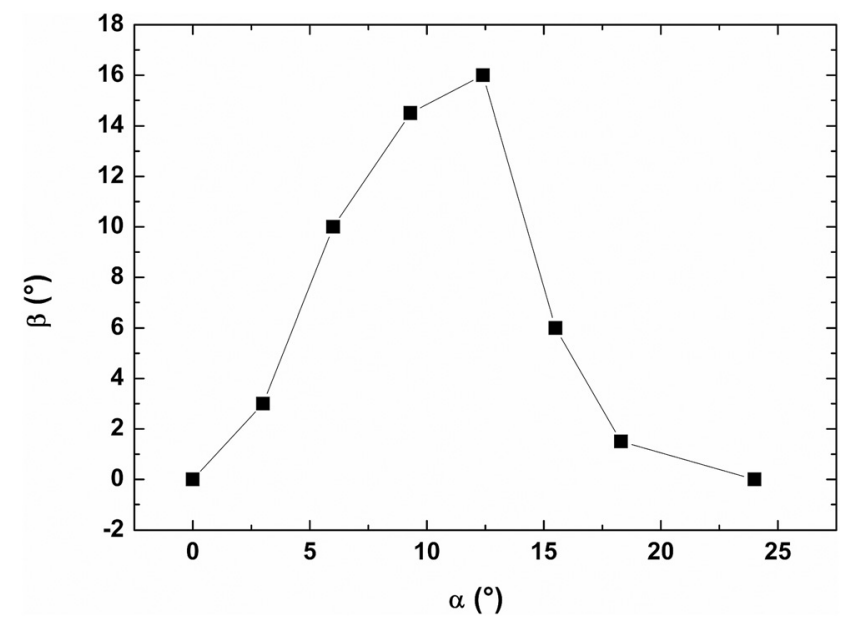

FIG. 8. Variation of the tilt $\beta$ of the c-axis as a function of the incident angle of the species $\alpha$. case of the shadowing effect (as indicated in the inset presented in this figure). ${ }^{33}$

\section{DISCUSSION}

The (001) or mixed (001) and (101) $\mathrm{ZnO}$ textures, tilted or not, were observed as a function of the position on the substrate, i.e., as a function of the oblique incidence $\alpha$ of the species. Generally, under oblique angle deposition at RT by thermal evaporation or sputtering, the film texture is inclined towards the incident species direction, due to the shadowing effect and limited atom diffusion. ${ }^{33-35}$ For the films grown by PLD at RT, we always observe an inclination of the c-axis in the forward direction, i.e., the reverse of the shadowing effect.

A possible explanation of this specific tilt of the (001) and (101)-oriented $\mathrm{ZnO}$ crystallites could be searched in the frame of the combined effects of the oblique angle deposition and variation of the ratio of fluxes $(\mathrm{O} / \mathrm{Zn})$ as a function of position. The tilt of these (001) and (101)-oriented crystallites is observed in zone II in which growth occurs under oblique incidence and with a $\mathrm{O} / \mathrm{Zn}$ ratio leading to the formation of oxygen deficient zinc oxide, i.e., under reducing conditions with a $\mathrm{Zn}$ excess. These two conditions are not present elsewhere in the films. In zone I, the incident species are mainly normal to the substrate, and despite the strong reducing character of the ratio of fluxes $(\mathrm{O} / \mathrm{Zn})$, a c-axis texture is observed. In zone III $\left(\alpha>25^{\circ}\right)$, the oblique incidence is present but the ratio of fluxes $(\mathrm{O} / \mathrm{Zn})$ is no longer reducing as it leads to a stoichiometric film, which presents a c-axis texture. Reducing growth conditions (Zn excess) and oblique angle deposition are thus both needed to observe the tilt of the (001) and (101)-oriented crystallites.

To explain the tilt of the (001) and (101)-oriented crystallites, the effect of stress during the growth has to be considered. Actually, residual stress plays an important role in the micro-structural evolution of thin films. ${ }^{36,37}$ In the case of PLD, the incident species possess a high kinetic energy, up to $100 \mathrm{eV}$ (Refs. 1 and 3) under typical conditions, and 

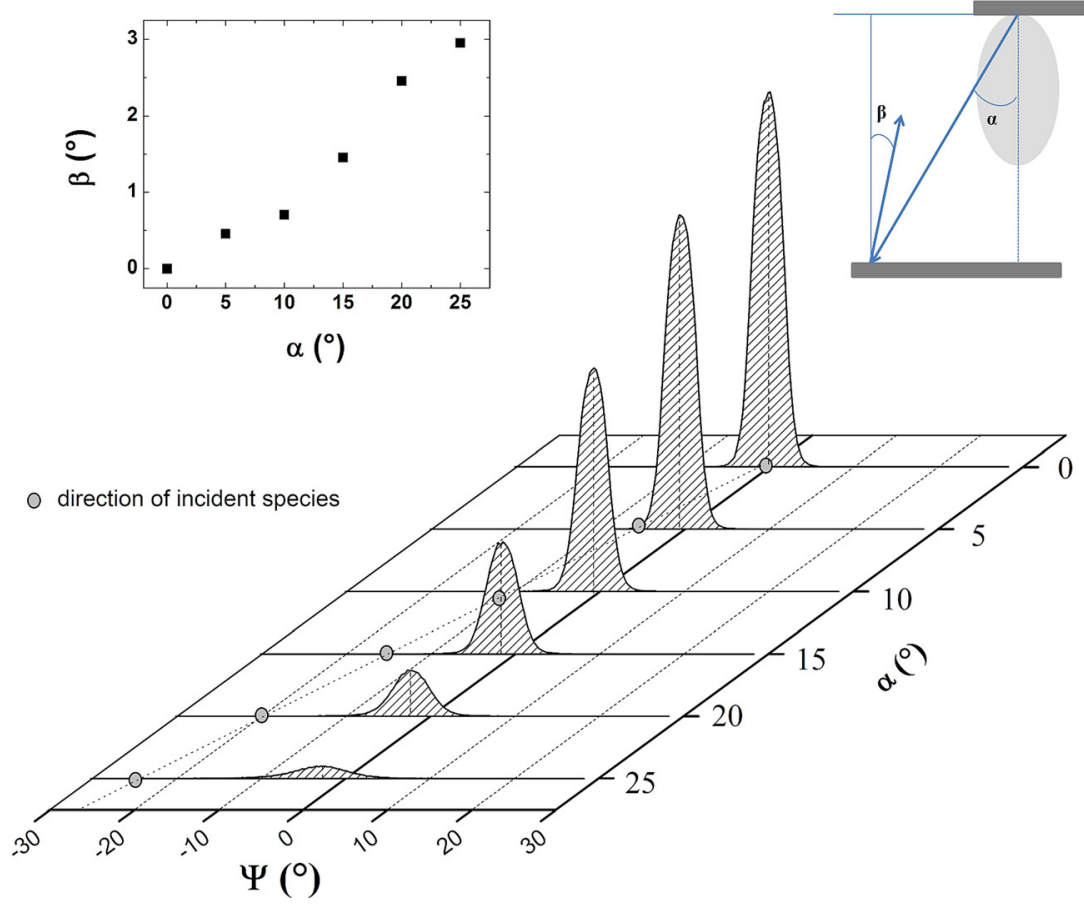

FIG. 9. $\Psi$ scan for the 002 reflection plane as a function of the incident angle of species $\alpha$ for a $\mathrm{ZnO}$ film grown at $500^{\circ} \mathrm{C}$ and $10^{-6}$ mbar. The inset shows schematically the respective directions of the incident species and the tilted c-axis. they irradiate the surface of the growing film. Under energetic bombardment conditions, a large compressive stress is observed during the growth of thin films, due to the atomic peening, which not only induces defects inside the crystallites but also promotes the incorporation of excess atoms in the grain boundaries. 36

In the present case, under normal incidence (zone I), the excess $\mathrm{Zn}$ atoms will be incorporated at the grain boundaries between c-axis oriented crystallites, leading thus to a broadening of the angular distribution of these crystallites. Under oblique incidence (zone II), the excess $\mathrm{Zn}$ atoms will be preferentially incorporated in the exposed side of the crystallites rather than in the shadowed side. This will give a tilt of the c-axis crystallites towards the shadowed side, i.e., in the direction opposite to the incident species with respect to the normal to the substrate. At larger incidence angle (zone III), two phenomena occur: (i) excess $\mathrm{Zn}$ atoms are not present (a stoichiometric film is formed in this zone) and (ii) the kinetic energy of the species decreases at large angles, ${ }^{38}$ i.e., decreasing thus the atomic peening effect. As a result, the usual c-axis texture is observed.

Similar observations have been recently reported. ${ }^{39,40}$ Indeed, oblique-angle sputtering of $\mathrm{ZnO}$ in a reducing atmosphere, i.e., a $20 \%$ hydrogen-argon gas mixture, led to the formation of "bent $\mathrm{ZnO}$ columns" in the direction opposite to the incident species. ${ }^{39}$ The TEM experiments revealed that the lattice bending phenomenon was accommodated by dislocation networks where the exposed side to the incident species of the column contains more dislocation than the shadowed side. Such a process leads to the columns bending towards the shadowed side, i.e., in the opposite direction to the incident species. This oblique angle deposition under argon-hydrogen reducing ambient has been used to grow highly oriented non-polar $\mathrm{ZnO}$ thin films on glass substrates. ${ }^{40}$ The microstructure of the non polar $\mathrm{ZnO}$ films consists in a stack of grains with the c-axis gradually rotated with increasing thickness from the normal to the nearly lateral direction with respect to the substrate surface. ${ }^{40}$ This "lattice bending phenomenon" could be described as the result of the stress induced by the oblique incident species (atomic peening) on the $\mathrm{ZnO}$ crystallites.

In the literature concerning $\mathrm{ZnO}$ films grown under oblique incidence, the results are rather confusing. Indeed, a columnar structure inclined towards the incident species has been reported in some cases, ${ }^{41}$ while $\mathrm{ZnO}$ films grown by DC sputtering were found to present their c-axis inclined in the forward direction (with a few degrees) with respect to the incident species. ${ }^{42}$ These contradictory results could be due to the differences in the nature (neutral or ionic) and kinetic energy of the incident species in the various deposition processes used for films growth. ${ }^{41}$ In particular, due the high kinetic energy of the species, the PLD growth presents similarities with the ion beam assisted deposition (IBAD) method. The IBAD process can lead to the growth of films with "unusual" texture, i.e., different from that deduced from surface free energy considerations. ${ }^{43}$ The changes in film texture in IBAD are explained by the preferential sputtering of differently oriented crystallites. ${ }^{44,45}$ It follows that the crystal directions able to reduce sputtering and lattice damages are the most open channeling directions, which limit the impinging ions-atoms collisions; they will therefore be favored in the growth. ${ }^{45}$ For $\mathrm{ZnO}$, the (001) oriented crystallites are very sensitive to ion irradiations, while the (100) and (110) axes are ion channeling directions, i.e., ion irradiation will induce (100) or (110) $\mathrm{ZnO}$ texture. ${ }^{46}$ In our work, we do not observe the (100) or (110) textures, whatever the oblique incidence $\alpha$ of the species, meaning that sputtering cannot explain our results.

The substrate temperature is an important parameter on the film microstructure. We only observe a behavior compatible with the shadowing effect for films grown at relatively high substrate temperature $\left(500^{\circ} \mathrm{C}\right)$ (see Fig. 9). However, in 
this case the tilted $\beta$ values are very small (only a few degrees) for oblique incidence up to $25^{\circ}$. The reason why would be that at $500^{\circ} \mathrm{C}$, the incident $\mathrm{Zn}$ species possess a high surface mobility that allows their migration on the film surface, which is generally very efficient to avoid the tilt of the column associated to the shadowing effect. ${ }^{34}$

Taking into account the results obtained in this work it comes that the methods used to obtain uniform film thickness and composition by PLD based on rotation and motion of the substrate with respect to the target could not necessarily lead to uniform crystalline orientation for large area $\mathrm{ZnO}$ films. At the best they will give a mean $\mathrm{ZnO}$ (001) orientation normal to the substrate, but with a very broad angular distribution. The film deposition at relatively high $\mathrm{T}$ may decrease these crystalline non-uniformities. This is certainly the reason why it was necessary to work at very high temperature $\left(1000{ }^{\circ} \mathrm{C}\right)$ to obtain uniform crystalline films on large scale. ${ }^{47}$ However, such a high deposition temperature would be a severe problem for a lot of devices involving such films. On the other hand, the approaches based on off-axis deposition would certainly give films with a uniform crystalline structure, but the related low deposition rate seems to preclude their common use in industrial context.

\section{CONCLUSIONS}

In addition to the non-uniformities in $\mathrm{ZnO}$ film thickness and stoichiometry as a function of position on the substrate, we have evidenced large non-uniformities in the crystalline lattice parameter and orientation in relation with the oblique incidence of the species emitted during PLD. Actually, (001) or mixed (001) and (101) $\mathrm{ZnO}$ textures, tilted or not, are observed as a function of the position on the substrate, i.e., as a function of the oblique incidence $\alpha$ of the species. As a matter of fact, for the film grown at $\mathrm{RT}$, the $\mathrm{ZnO}$-axis presents an inclination angle which cannot be described by the well-known shadowing effect. The main parameters governing the unexpected tilt of the (001) and (101)-oriented crystallites are the oblique incidence of the species combined to the ratio of fluxes of oxygen and $\mathrm{Zn}$ species, which both depends upon the position on the substrate.

This work demonstrates that all the methods used to obtain uniform film thickness and composition by PLD and which are mainly based on rotation and motion of the substrate with respect to the target will not necessarily lead to films with uniform crystalline orientation. At the best they will give a mean $\mathrm{ZnO}$ (001) orientation normal to the substrate, but with a broad angular distribution, i.e., a quasi polycrystalline film will be obtained. The structural nonuniformities have to be taken into account in the next generation of PLD systems for large area deposition. New approaches are needed to obtain using a moderate growth temperature, thin films with an acceptable deposition rate and a perfect crystalline uniformity on large dimension.

\section{ACKNOWLEDGMENTS}

M. Nistor would thank a grant of the Romanian National Authority for Scientific Research, CNCS-UEFISCDI, Project No. PN-II-ID-PCE-2011-3-0566.

\section{APPENDIX: INTERPRETATION OF $\Psi$ SCANS}

In this work, the $\psi$ scans recorded for the 002 diffraction line show the presence of a main peak located at a $\beta$ angle depending of the oblique incidence $\alpha$, with two other low intensity peaks located at about $\beta+60^{\circ}$ (Fig. 7). It follows that the main peak located at $\beta$ is due to the (001)-oriented $\mathrm{ZnO}$ crystallites $\left(\mathrm{C}_{(001)}\right)$ tilted by a $\beta$ angle. Moreover, the presence of both (001) and (101)-oriented $\mathrm{ZnO}$ crystallites in the films has been established (Fig. 2). As the angle between the (002) and (101) planes of the $\mathrm{ZnO}$ wurtzite structure is equal to $61.6^{\circ}$, the assumption that both (001) and (101)-oriented crystallites are tilted by the same $\beta$ angle leads to the conclusion that the two low intensity peaks correspond to the (002)

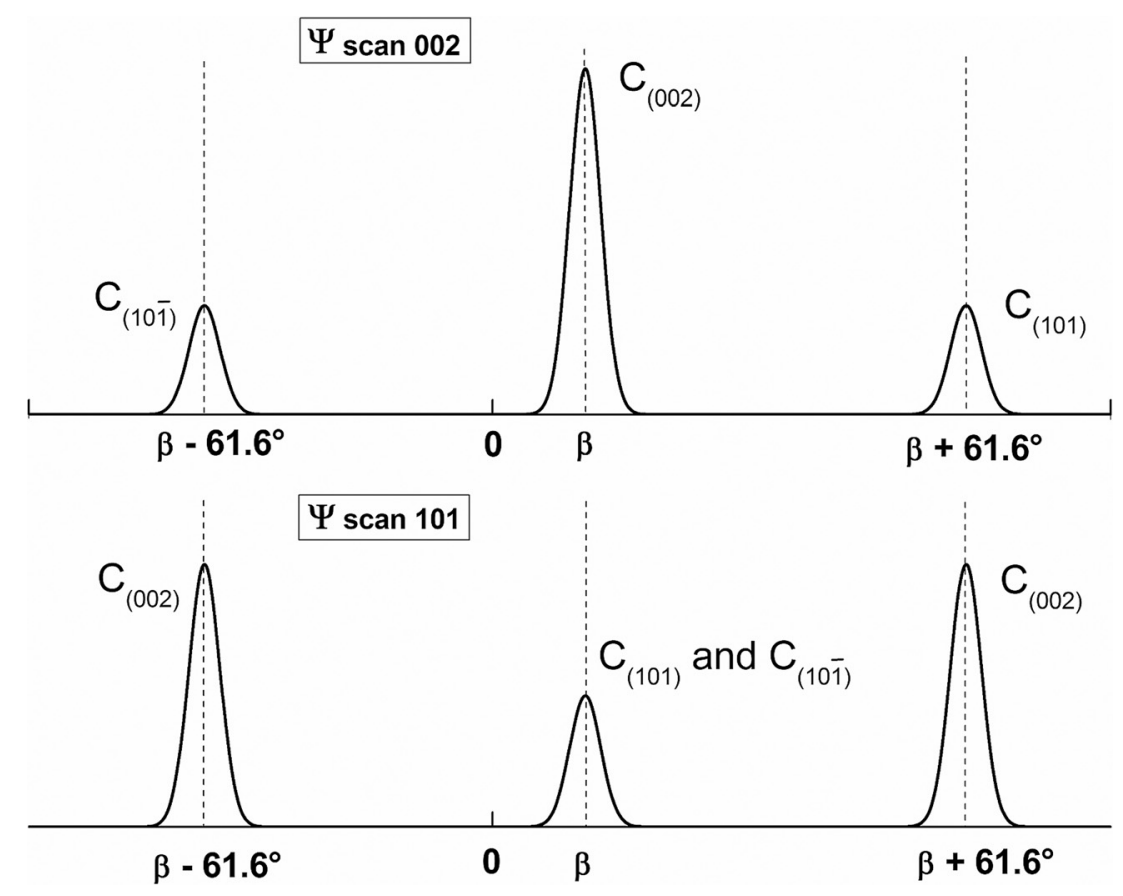

FIG. 10. Schematic representation of the contributions of the (001) and (101)-oriented $\mathrm{ZnO}$ crystallites to $\Psi$ scans for the 002 (a) and 101 (b) reflection lines. 


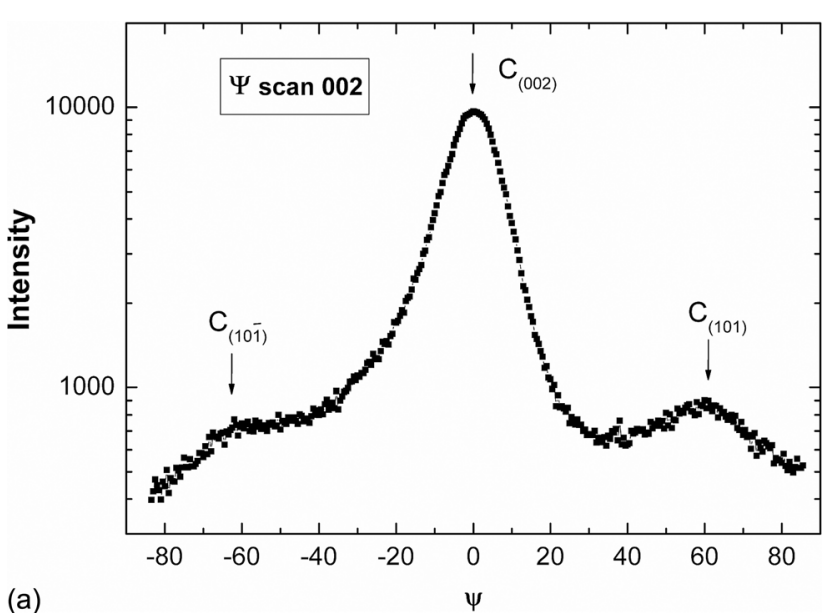

(a)

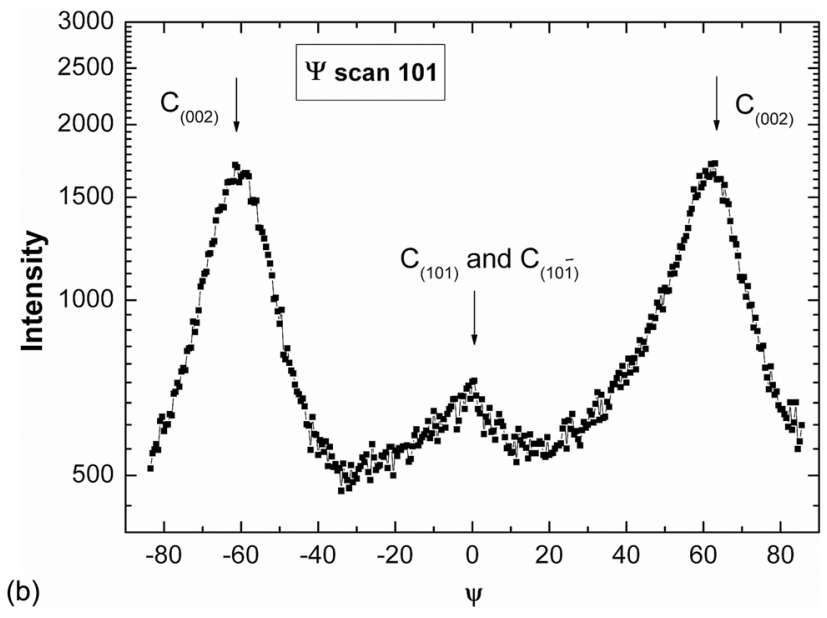

FIG. 11. $\Psi$ scans for the 002 (a) and 101 (b) reflection lines, for an incident angle of the species $\alpha$ equal to $20^{\circ}$.

planes of the (101) and (10-1) $\mathrm{ZnO}$ crystallites $\left(\mathrm{C}_{(101)}\right.$ and $\left.\mathrm{C}_{(10-1)}\right)$ tilted by the $\beta$ angle, as it is schematized in Fig. 10(a) in which these peaks are located at $\beta \pm 61.6^{\circ}$.

In this frame, it comes that a $\Psi$ scan of the $101 \mathrm{ZnO}$ diffraction line will show a peak located at $\beta$, corresponding to the $\mathrm{C}_{(101)}$ and $\mathrm{C}_{(10-1)} \mathrm{ZnO}$ crystallites tilted by an angle $\beta$, and two peaks located at $\beta \pm 61.6^{\circ}$, corresponding to the (101) planes of the $\mathrm{C}_{(001)}$ crystallites tilted by an angle $\beta$. To check this approach, $\Psi$ scans of the 002 and 101 diffraction lines were recorded for an oblique incidence $(\alpha=20)$, and they are shown in Figs. 11(a) and 11(b). The comparison of these two $\Psi$ scans shows the coincidence of both the "central" 002 and 101 peaks, as well as the coincidence of the 002 and 101 peaks located at $\pm 61.6^{\circ}$ from the "central" peaks. We can thus conclude that the $\Psi$ scans presented in Fig. 7 are due to the presence of both (001) and (101)-oriented crystallites tilted by a $\beta$ angle with respect to the normal to the substrate.

${ }^{1}$ Pulsed Laser Deposition of Thin Films, edited by R. Weason (WileyIntersciences, 2007).

${ }^{2}$ R. E. Muenchhausen, K. M. Hubbard, S. Foltyn, R. C. Esther, N. S. Nogar, and C. Jenkins, Appl. Phys. Lett. 56, 578 (1990).

${ }^{3}$ N. Pryds, J. Schou, and S. Linderoth, Appl. Surf. Sci. 253, 8231 (2007).

${ }^{4}$ S. I. Anisimov, D. Bauerle, and B. S. Lukyanchuk, Phys. Rev. B 48, 12076 (1993).

${ }^{5}$ B. Toftmann, J. Schou, and J. G. Lunney, Phys. Rev. B 67, 104101 (2003).
${ }^{6}$ S. Amoruso, B. Toftmann, and J. Schou, Phys. Rev. E 69, 056403 (2004). ${ }^{7}$ M. Nistor, F. Gherendi, and N. B. Mandache, Appl. Surf. Sci. 258, 9274 (2012).

${ }^{8}$ E. Millon, M. Nistor, C. Hebert, Y. Davila, and J. Perrière, J. Mater. Chem. 22, 12179 (2012).

${ }^{9}$ J. A. Greer, "History and current status of commercial pulsed-laser deposition equipment," J. Phys. D: Appl. Phys. 47, 034005 (2014).

${ }^{10}$ L. Egerhazi, Z. S. Geretovsky, T. Szorenyi, and F. Bari, Appl. Surf. Sci. 257, 5324 (2011).

${ }^{11}$ T. C. Droubay, L. Qiao, T. C. Kaspar, M. H. Engelhard, V. Shutthananandan, and S. A. Chambers, Appl. Phys. Lett. 97, 124105-124107 (2010).

${ }^{12}$ A. Ohtomo and A. Tsukazaki, Semicond. Sci. Technol. 20, S1 (2005).

${ }^{13}$ S. J. Pearton, D. P. Norton, K. Ip, Y. W. Heo, and T. Steiner, Prog. Mater. Sci. 50, 293 (2005).

${ }^{14}$ Y. Thimont, J. Clatot, M. Nistor, C. Labrugere, and A. Rougier, Sol. Energy Mater. Sol. Cells 107, 136-141 (2012).

${ }^{15}$ N. Jedrecy, H. J. von Bardeleben, and D. Demaille, Phys. Rev. B 80, 205204 (2009).

${ }^{16}$ M. Morcrette, A. Gutierrez-Llorente, W. Seiler, J. Perriere, A. Laurent, and P. Barboux, J. Appl. Phys. 88, 5100 (2000).

${ }^{17}$ R. K. Singh and J. Narayan, Phys. Rev. B 41, 8843 (1990).

${ }^{18} \mathrm{~S}$. Amoruso, M. Armenante, R. Bruzzese, N. Spinelli, R. Velotta, and X. Wang, Appl. Phys. A 71, 215 (2000).

${ }^{19}$ X. Wang, S. Amoruso, R. Bruzzese, N. Spinelli, A. Torfora, R. Verlotta, C. Ferdeghini, G. Grassano, and W. Ramadan, Chem. Phys. Lett. 353, 1 (2002).

${ }^{20}$ A. Perea, J. Gonzalo, C. N. Afonso, C. Vivien, and C. Leborgne, Appl. Phys. A 69, S141 (1999).

${ }^{21}$ J. Gonzalo, C. N. Afonso, and J. Perrière, Appl. Phys. Lett. 67, 1325 (1995).

${ }^{22}$ L. Cossarutto, N. Chaoui, E. Millon, J. F. Muller, J. Lambert, and M. Alnot, Appl. Surf. Sci. 126, 352 (1998).

${ }^{23}$ E. LeBoulbar, E. Millon, J. Mathias, C. Boulmer-Leborgne, M. Nistor, F. Gherendi, N. Sbaï, and J. B. Quoirin, Appl. Surf. Sci. 257, 5380 (2011).

${ }^{24}$ H.-J. Ko, T. Yao, Y. Chen, and S. K. Hong, J. Appl. Phys. 92, 4354 (2002).

${ }^{25}$ V. Craciun, R. K. Singh, J. Perriere, J. Spear, and D. Craciun, J. Electrochem. Soc. 147, 1077 (2000).

${ }^{26}$ M. Nistor, N. B. Mandache, J. Perriere, C. Hebert, F. Gherendi, and W. Seiler, Thin Solid Films 519, 3959 (2011).

${ }^{27}$ P. W. Tasker, J. Phys. C: Solid State Phys. 12, 4977 (1979).

${ }^{28}$ S. Tricot, M. Nistor, E. Millon, C. Boulmer-Leborgne, N. B. Mandache, J. Perriere, and W. Seiler, Surf. Sci. 604, 2024 (2010).

${ }^{29}$ Y. E. Lee, J. B. Lee, Y. J. Kim, H. K. Yang, J. C. Park, and H. J. Kim, J. Vac. Sci. Technol., A 14, 1943-1948 (1996).

${ }^{30}$ B. J. Jin, S. H. Bae, S. Y. Lee, and S. Im, Mater. Sci. Eng., B 71, 301 (2000).

${ }^{31}$ M. Nistor, W. Seiler, C. Hebert, E. Matei, and J. Perrière, Appl. Surf. Sci. 307, 455 (2014).

${ }^{32}$ O. Bierwagen, M. E. White, M. Y. Tsai, and J. S. Speck, Appl. Phys. Lett. 95, 262105 (2009).

${ }^{33}$ R. N. Tait, T. Smy, and M. J. Brett, Thin Solid Films 226, 196 (1993).

${ }^{34}$ D. Zemsky, R. Shneck, P. J. Dagdigian, and I. Bar, J. Appl. Phys. 102, 104309 (2007).

${ }^{35}$ D. J. Paritosh and J. Srolovitz, J. Appl. Phys. 91, 1963 (2002).

${ }^{36}$ L. E. Koutsokeras and G. Abadias, J. Appl. Phys. 111, 093509 (2012).

${ }^{37}$ S. K. Sharma and D. Y. Kim, Curr. Appl. Phys. 13, 1874 (2013).

${ }^{38}$ B. Toftmann, J. Schou, and S. Canulescu, Appl. Surf. Sci. 278, 273 (2013).

${ }^{39}$ J. H. Huang, C. Y. Chen, Y. F. Lai, Y. U. Shih, Y. C. Lin, J. H. He, and C. P. Liu, Cryst. Growth Des. 10, 3297 (2010).

${ }^{40}$ Y. F. Lai, J. H. Huang, Y. C. Chen, C. P. Liu, and Y. W. Yang, J. Eur. Ceram. Soc. 33, 1809 (2013).

${ }^{41}$ Y. E. Lee, S. G. Kim, Y. S. Kim, and H. J. Kim, J. Vac. Sci. Technol., A 15, 1194 (1997)

${ }^{42}$ I. Cerven, T. Lacko, I. Novotny, V. Tvarozek, and M. Harvanka, J. Cryst. Growth 131, 546 (1993).

${ }^{43}$ T. Yanagitani and M. Kiuchi, Surf. Coat. Technol. 206, 816 (2011).

${ }^{44}$ W. Ensinger, Nucl. Instrum. Methods Phys. Res., Sect. B 106, 142 (1995).

${ }^{45}$ L. Dong and D. J. Srolovitz, Appl. Phys. Lett. 75, 584 (1999).

${ }^{46}$ T. Yanagitani and M. Kiuchi, J. Appl. Phys. 102, 044115 (2007).

${ }^{47}$ H. Yang, W. Wang, Z. Liu, and G. Li, J. Phys. D: Appl. Phys. 46, 105101 (2013). 